Article

\title{
Landauer's Principle and Divergenceless Dynamical Systems
}

\section{Claudia Zander ${ }^{1}$, Angel Ricardo Plastino ${ }^{1,2,3}$, Angelo Plastino ${ }^{3, \star}$, Montserrat Casas ${ }^{4}$ and $^{-}$} Sergio Curilef ${ }^{5}$

${ }^{1}$ Physics Department, University of Pretoria, Pretoria 0002, South Africa;

E-Mail: Claudia.Zander@up.ac.za

${ }^{2}$ Instituto de Física Teórica y Computacional Carlos I, Facultad de Ciencias, Universidad de Granada, Granada, Spain; E-Mail: arplastino@maple.up.ac.za

${ }^{3}$ National University La Plata, UNLP-CREG-CONICET, Casilla de Correos 727, 1900 La Plata, Argentina

${ }^{4}$ Departament de Física, Universitat de les Illes Balears, Mallorca, Spain;

E-Mail: Montse.Casas@uib.es

${ }^{5}$ Departamento de Física, Universidad Católica del Norte, Antofagasta, Chile; E-Mail: scurilef@ucn.cl

* Author to whom correspondence should be addressed; E-Mail: plastino@ sinectis.com.ar.

Received: 17 August 2009 / Accepted: 15 September 2009 / Published: 13 October 2009

\begin{abstract}
Landauer's principle is one of the pillars of the physics of information. It constitutes one of the foundations behind the idea that "information is physical". Landauer's principle establishes the smallest amount of energy that has to be dissipated when one bit of information is erased from a computing device. Here we explore an extended Landauerlike principle valid for general dynamical systems (not necessarily Hamiltonian) governed by divergenceless phase space flows.
\end{abstract}

Keywords: information physics; Landauer principle; entropy; dynamical systems

Classification: PACS 89.70.+c, 02.50.-r, 05.20.Gg

\section{Introduction}

The physics of information constitutes an active research field that has been the focus of considerable attention in recent times [1-10]. Due to seminal results generated by these research efforts the physical 
reality of information is by now generally acknowledged. In this regard, the ultimate performance limits imposed by the laws of physics on any real device that processes or transmits information are starting to be understood $[6,11]$. On the other hand, several theoretical developments indicate that the concept of information is essential for understanding the basic fabric of the physical world [1-6]. Tools inspired by information-theoretical ideas, such as the maximum entropy (maxent) principle [12-14] have been successfully applied to the study of several physical scenarios. Last, but certainly not least, the ideas and methods from the physics of information lead to important points of contact between physics and biology. In fact, information processing is clearly at the very heart of biology and has been appropriately dubbed the "touchstone of life" [15].

Landauer's principle is one of the most fundamental results in the physics of information. It constituted a historical landmark in the development of the field by directly connecting information processing with conventional physical quantities [16]. Most remarkably, it played a prominent role in the final defeat of Maxwell's demon [6]. Landauer's principle states that there is a minimum amount of energy that has to be dissipated, on average, when erasing a bit of information in a computing device working at absolute temperature $T$. This minimum energy is equal to $k T \ln 2$, where $k$ is Boltzmann's constant [17-20]. Landauer's principle has profound implications as it allows for novel, physically motivated derivations of several important results in classical and quantum information theory [21]. Moreover, it proved to be a powerful heuristic tool for establishing new links between, or obtaining new derivations of, fundamental aspects of thermodynamics and other areas of physics [22].

It is fair to say that most derivations of Landauer's principle can be regarded as semi-phenomenological, since they are based on a direct application of the second principle of thermodynamics. However, derivations based upon dynamical principles have also been advanced. They assume that the systems under consideration are governed by a Hamiltonian dynamics and are in thermal equilibrium, implying that they can be described by Gibb's canonical distributions. In view of the fundamental character of Landauer's principle, however, it is highly desirable to explore extensions of it applicable to systems governed by more general kinds of dynamics. These developments are inscribed within the more general program of extending the methods of statistical mechanics to non-Hamiltonian systems [23, 24]. Of special relevance is the class of dynamical systems with divergenceless phase-space flows, that include Hamiltonian systems as particular members. Divergenceless systems are characterized by the remarkable property that their dynamics preserves information. There are interesting divergenceless dynamical systems in physics, theoretical biology and other areas that are not Hamiltonian, or that have their most natural description in terms of a non-canonical set of variables. For example, the Lotka-Volterra predator-prey systems [26, 27] and the Nambu systems [28] share the vanishing divergence property. The Lotka-Volterra predator-prey systems constitute some of the most important dynamical systems considered in theoretical biology [26]. Nambu systems have been the focus of considerable research activity (see [29-33] and references therein). The main difference between Hamiltonian systems and Nambu systems is that, while the dynamics of a Hamiltonian system is governed by one single phase-space function (the Hamiltonian function) the dynamics of a Nambu system is governed by a set of $N(N \geq 2)$ such functions or "Hamiltonians" [28]. The dynamics of Nambu systems can be formulated in terms of Poisson-like brackets involving, in general, more than two functions. In the case of a Nambu system with $N$ "Hamiltonians" the time derivative of a general phase-space function $A$ is given by an appropriate $(N+1)$-bracket de- 
pending on the function $A$ and on the $N$ Hamiltonians. Unlike Hamiltonian systems, Nambu systems can be defined on phase-spaces with an odd number of dimensions. The Nambu dynamical structures arise in a natural way in several contexts. For instance, Nambu dynamics has been applied to the relativistic dynamics of charged spinning particles [32], and to some hydrodynamical type systems [33].

Information processing can be realized in various physical settings. Indeed, one of the main ideas behind the physics of information and computation is that every physical system (even the whole universe) can be construed as an information processing system. Consequently, it is of considerable interest to extend the fundamental principles of the physics of information to more general scenarios. These more general situations may encompass compelling examples of physical realizations of information processes such as, for instance, those related to biology. Indeed, biological systems process information at molecular, cellular, and higher levels [15].

The aim of the present work is to explore extensions of Landauer's principle to the aforementioned more general scenarios. We shall derive a Landauer-like principle for dynamical systems with divergenceless phase-space flows. This result establishes a direct connection (within the alluded dynamical context) between an information-processing operation, on the one hand, and changes in the mean value of a relevant dynamical quantity, on the other one. In the particular case of the usual Hamiltonian scenario our generalized result reduces to the standard Landauer principle. There is much to be gained from considering Landauer-like principles within the abovementioned general dynamical settings. Besides opening a range of possible new applications, this line of enquiry contributes to a deeper understanding of the main dynamical and information-theoretical ingredients at play behind Landauer's principle. The paper is organized as follows: in Section II we derive a generalized Landauer-like principle valid for divergenceless systems. Section III attempts to provide a further extension of this result utilizing the superstatistical formalism. Some conclusions are given in Section IV.

\section{An Extension of Landauer's Principle to Divergenceless Dynamical Systems}

In this section we are going to formulate a generalization of Landauer's principle appropriate for dynamical systems with vanishing divergence. We are going to consider a composite system $A+B$ compounded by an information "storing device" $A$ and an "environment" $B$. The composite system will be described by an appropriate ensemble probability density describing the probability of finding the system in different regions of phase space. Generalizing the standard Hamiltonian scenario, the erasure of one bit of information from the storing device $A$ will be identified with a dynamical process (described by an appropriate Liouville equation) where the Gibbs-Shannon entropy of the marginal probability density corresponding to the device $A$ decreases by $k \ln 2$ (here $k$ is an appropriate constant defining the units in which the entropy is measured; in the standard Hamiltonian statistical mechanics setting $k$ stands for Boltzmann's constant). In the Hamiltonian case the device $A$ is modelled as a particle moving in a one-dimensional double-well potential, with the left and right wells representing the two possible states of the classical bit ("0" and " 1 "). Before erasure the marginal probability density describing the particle is such that the particle is equally likely to be in either well. This corresponds to a situation where the two states of the bit are equally probable. After erasure the bit is set to one of its possible states (say, state " 0 ”), the particle's marginal probability density is concentrated in one well, and its entropy decreases by an amount $k \ln 2$. In the more general setting that we are going to explore we shall interpret the 
decrease in $k \ln 2$ of the entropy of subsystem $A$ as the most fundamental characterization of a process corresponding to the erasure of one bit of information from $A$. This entropy reduction corresponds to a decrease of the "spread" of the phase space distribution of $A$ and, consequently also a decrease of the amount of information that can be encoded in the precise location of system $A$ in phase space.

\subsection{Divergenceless Dynamical Systems}

Let us consider a dynamical system

$$
\dot{\boldsymbol{z}}=\boldsymbol{w}(\boldsymbol{z}), \quad \boldsymbol{z}, \boldsymbol{w} \in \mathcal{R}^{N}
$$

where the vector $z$ represents a point in the system's phase space. The phase space variables are separated in two-subsets,

$$
z=(\boldsymbol{x}, \boldsymbol{y})
$$

with

$$
\begin{array}{llll}
\boldsymbol{x} & \in \mathcal{R}^{N_{1}}, & \\
\boldsymbol{y} & \in \mathcal{R}^{N_{2}}, & N_{1}+N_{2}=N
\end{array}
$$

where $\boldsymbol{x}$ stands for the phase space coordinates of the "information storage device" $A$ and $\boldsymbol{y}$ denotes the phase space coordinates of the environment $B$.

The dynamical equations of motion of the composite $A+B$ can be written as

$$
\begin{aligned}
\dot{\boldsymbol{x}} & =\boldsymbol{u}(\boldsymbol{x}, \boldsymbol{y}) \\
\dot{\boldsymbol{y}} & =\boldsymbol{v}(\boldsymbol{x}, \boldsymbol{y})
\end{aligned}
$$

where $\boldsymbol{w}=(\boldsymbol{u}, \boldsymbol{v})$ is a divergence-free phase-space flow. That is,

$$
\nabla \cdot \boldsymbol{w}=\left(\sum_{i=1}^{N_{1}} \frac{\partial u_{i}}{\partial x_{i}}\right)+\left(\sum_{j=1}^{N_{2}} \frac{\partial v_{j}}{\partial y_{j}}\right)=0
$$

where the $N$-dimensional $\nabla$-operator is defined in the standard way,

$$
\nabla=\left(\frac{\partial}{\partial x_{1}}, \ldots, \frac{\partial}{\partial x_{N_{1}}}, \frac{\partial}{\partial y_{1}}, \ldots, \frac{\partial}{\partial y_{N_{2}}}\right)
$$

We are going to consider divergenceless dynamical systems (4) admitting a constant of motion $I(\boldsymbol{x}, \boldsymbol{y})$,

$$
\frac{d I}{d t}=\left(\sum_{i=1}^{N_{1}} \frac{\partial I}{\partial x_{i}} u_{i}\right)+\left(\sum_{j=1}^{N_{2}} \frac{\partial I}{\partial y_{j}} v_{j}\right)=0
$$

Hamiltonian systems fulfill condition (5) and admit the Hamiltonian itself as a constant of motion, but there are other interesting systems compliant with (5) and admitting an integral of motion $I$. Indeed, these two fundamental features allow for deriving many aspects of the canonical statistical mechanical 
formalism without recourse to the detailed structure of standard Hamiltonian dynamics [24, 25]. Among others, the Lotka-Volterra predator-prey systems [26, 27] and the Nambu systems [28-33] share the vanishing divergence property and admit an integral of motion.

If the system $A$ is to behave as a proper "information storage device", it is reasonable to assume that before and after the information erasure process, the systems $A$ and $B$ are only weakly coupled. In fact, as far as the storage of information is concerned, the effect of this "weak coupling" is assumed to be negligible before and after the erasure process (a system that "preserves" information should not interact too strongly with the environment). That is, we assume that in Equation (4), $\boldsymbol{u}$ depends weakly on $\boldsymbol{y}$ and $\boldsymbol{v}$ depends weakly on $\boldsymbol{x}$. Consistent with the weak coupling between systems $A$ and $B$, we are also going to assume that before and after the information erasure the integral of motion $I$ adopts the additive form

$$
I=D(\boldsymbol{x})+C(\boldsymbol{y}) .
$$

The vector fields $\boldsymbol{u}$ and $\boldsymbol{v}$ are assumed to be time independent before and after the erasure process (that is, the dynamical system (4) is autonomous), but $\boldsymbol{u}$ and $\boldsymbol{v}$ may depend explicitly on time during the erasure process. Consequently, during the erasure process the quantity $I$ may not be conserved, but it is strictly conserved before and after that process. Notice, however, that the conservation of $I$ does not imply that $D$ and $C$ are individually conserved. Even the weak coupling between the systems $A$ and $B$ allows for changes in $C$ and $D$, but keeping their sum constant. In the limit case of no interaction between systems $A$ and $B$, the quantities $D$ and $C$ become individual integration constants of these two systems, respectively. This situation is similar to the one considered (in connection with the energy) in standard statistical mechanics when discussing a system weakly coupled to a heat bath. The divergenceless property (5), which is the most important feature characterizing the dynamical systems that are considered here, and the main ingredient in the derivation of the extended Landauer's principle, is assumed to hold all the time.

A time dependent statistical ensemble of systems evolving according to the equations of motion (4) is described by a phase-space probability density $F(\boldsymbol{x}, \boldsymbol{y}, t)$ governed by Liouville's equation,

$$
\frac{\partial F}{\partial t}+\nabla \cdot(F \boldsymbol{w})=0
$$

which, due to the divergenceless condition $\nabla \cdot \boldsymbol{w}=0$ reduces to

$$
\frac{\partial F}{\partial t}+\boldsymbol{w} \cdot \nabla F=0
$$

The Boltzmann-Gibbs-Shannon information measure is given by

$$
S[F]=-k \int F \ln F d \Omega
$$

where $k$ is an appropriate constant determining the units in which $S$ is measured and $d \Omega$ is the phasespace volume element. The time dependence of the Boltzmann-Gibbs-Shannon measure evaluated on a time dependent solution of Liouville's equation complies with (see [31] and references therein)

$$
\frac{d S}{d t}=\int F(\nabla \cdot \boldsymbol{w}) d \Omega=\langle\nabla \cdot \boldsymbol{w}\rangle
$$


implying that in the case of divergenceless systems we have

$$
\frac{d S}{d t}=0
$$

This equation summarizes one of the most important features of the behavior of divergenceless dynamical systems: the conservation of information. If, following Jaynes, we interpret $S[F]$ as a measure of our lack of knowledge of the precise state of the system, then equation (13) means that the amount of "missing" knowledge is constant in time. This information-preserving character of dynamical evolution is one of the most important features of the basic laws of nature, both in the classical and in the quantum regimes. Not only at the fundamental level is divergenceless dynamics relevant but also, in some cases, in connection with the phenomenological description of higher-level systems in physics, biology and other fields.

\section{2. $\quad$ Extended Landauer-Like Principle}

As part of the program of exploring some basic aspects of the physics of information within general dynamical settings, we here extend Landauer's principle to systems described by divergenceless phase space flows.

Prior to erasure the composite system is described by the ensemble distribution $F_{\text {initial }}=F_{\text {initial }}(\boldsymbol{x}, \boldsymbol{y})$ where, as already said, $\boldsymbol{x}$ and $\boldsymbol{y}$ represent the complete sets of phase space variables of the storage device $A$ and the environment $B$, respectively. After erasure the composite $A+B$ is given by the final distribution $F_{\text {final }}=F_{\text {final }}(\boldsymbol{x}, \boldsymbol{y})$. The concomitant marginal distributions read $F_{\text {initial/final }}^{(A)}=F_{\text {initial/final }}^{(A)}(\boldsymbol{x})=$ $\int F_{\text {initial/final }} d \Omega^{(B)}$ and $F_{\text {initial/final }}^{(B)}=F_{\text {initial/final }}^{(B)}(\boldsymbol{y})=\int F_{\text {initial/final }} d \Omega^{(A)}$, where $d \Omega^{(A)}$ and $d \Omega^{(B)}$ denote the phase space volume elements associated with the storing device and the environment, respectively. The total volume element of the composite system is $d \Omega^{(A+B)}=d \Omega^{(A)} d \Omega^{(B)}$. The erasure process starts with an initial distribution of the form

$$
F_{\text {initial }}(\boldsymbol{x}, \boldsymbol{y} ; \lambda)=\frac{e^{-\frac{\lambda}{k}(D(\boldsymbol{x})+C(\boldsymbol{y}))}}{Z^{(A)}(\lambda) Z^{(B)}(\lambda)}
$$

where $I=D(\boldsymbol{x})+C(\boldsymbol{y})$ denotes some appropriate, conserved dynamical quantity (see Equation (8)). $Z^{(A)}(\lambda)$ and $Z^{(B)}(\lambda)$ are the accompanying partition functions of the bit-storage device (system $A$ ) and the "environment" (system $B$ ), respectively given by

$$
\begin{aligned}
& Z^{(A)}(\lambda)=\int e^{-\frac{\lambda}{k} D(\boldsymbol{x})} d \Omega^{(A)} \\
& Z^{(B)}(\lambda)=\int e^{-\frac{\lambda}{k} C(\boldsymbol{y})} d \Omega^{(B)}
\end{aligned}
$$

We assume that both $D(\boldsymbol{x})$ and $C(\boldsymbol{y})$ comply with all the properties required for the convergence of $Z^{(A)}(\lambda)$ and $Z^{(B)}(\lambda)$. In particular, we assume that $D(\boldsymbol{x})$ and $C(\boldsymbol{y})$ are bounded from below. In the standard Hamiltonian case we have that $D=H_{A}$ and $C=H_{B}$ are the Hamiltonians of the information storage device and the environment (heat bath), respectively, and the initial distribution (14) corresponds to the Gibbs canonical distribution describing thermal equilibrium at temperature $T=1 / \lambda$. The initial distribution is also a maximum entropy distribution that maximizes the entropy under the constraint 
given by the mean value of $\langle I\rangle=\langle D+C\rangle$. Before the erasure process (when the dynamics of the $A+B$ system is autonomous and the quantity $I$ is conserved) this maxent distribution constitutes a stationary solution of Liouville's equation. This stationary maxent density can be regarded, according to information theoretical interpretation of equilibrium statistical mechanics, as the natural one for describing an equilibrium situation.

After erasure, the composite system, i.e., storage device plus heat bath, has evolved into a new state whose final distribution $F_{\text {final }}=F_{\text {final }}(\boldsymbol{x}, \boldsymbol{y} ; \lambda)$ yields a marginal distribution of the storage device that verifies:

$$
S\left[F_{\text {initial }}^{(A)}\right]=S\left[F_{\text {final }}^{(A)}\right]+k \ln 2
$$

In other words, the erasure of a single bit of memory implies a decrease of $(k \ln 2)$ in the entropy of the storage device.

The Lagrange multiplier $\lambda$ describing the initial distribution can be seen as a parameter that characterizes a family of different realizations of the erasure process $F_{\text {initial }}(\boldsymbol{x}, \boldsymbol{y} ; \lambda) \rightarrow F_{\text {final }}(\boldsymbol{x}, \boldsymbol{y} ; \lambda)$. Each realization is associated with a particular time-dependent solution of Liouville's equation, $\frac{\partial F}{\partial t}+\nabla \cdot(F \boldsymbol{w})=$ 0 . Notice, however, that the total ensemble distribution is not required to have the canonical form throughout the erasure process, particularly, not after the erasure process has finalized.

Before and after erasure the total entropies of the composite system are related by

$$
\begin{aligned}
S\left[F_{\text {initial }}\right] & =S\left[F_{\text {initial }}^{(A)}\right]+S\left[F_{\text {initial }}^{(B)}\right]=S\left[F_{\text {final }}\right] \\
& \leq S\left[F_{\text {final }}^{(A)}\right]+S\left[F_{\text {final }}^{(B)}\right]
\end{aligned}
$$

In (17) the first equality represents the additivity of Shannon's entropy for the initial canonical distribution (which is factorizable) and the second equality is the conservation of the total entropy in the course of Liouville's evolution. The inequality, in contrast, is due to the sub-additivity of Shannon's entropy [34]. This inequality becomes an equality if the final joint probability density describing the composite $A+B$ is factorizable. In the general case, however, when the systems $A$ and $B$ are correlated after the erasure process, we have a strict inequality in (17).

Combining (16) with (17) leads to

$$
S\left[F_{\text {final }}^{(B)}\right]-S\left[F_{\text {initial }}^{(B)}\right] \geq k \ln 2
$$

The maxent probability density can be characterized either as the probability density of maximum entropy $S$ for a given value of $\langle C\rangle$ or, alternatively, as the probability density of minimum $\langle C\rangle$ for a given $S$. Therefore, the initial and final mean values of $C(\boldsymbol{y})$ verify

$$
\langle C\rangle_{\text {final }}-\langle C\rangle_{\text {initial }} \geq\langle C\rangle_{\text {final }}^{(\text {(canonical) }}-\langle C\rangle_{\text {initial }}
$$

where $\langle C\rangle_{\text {final }}^{\text {(canonical) }}$ is the value of $\langle C\rangle$ associated with a maxent distribution having the same value of the entropy as $F_{\text {final }}^{(B)}$. Now, we have the following relations (which, replacing $H$ for $C$, coincide with well known relationships satisfied by Gibbs canonical distribution):

$$
d\langle C\rangle / d S=1 / \lambda>0
$$




$$
d^{2}\langle C\rangle / d S^{2}=-\left(1 / \lambda^{2}\right)(d \lambda / d S)>0
$$

Therefore,

$$
\begin{aligned}
\langle C\rangle_{\text {final }}^{(\text {canonical })}-\langle C\rangle_{\text {initial }} & \geq\left[S_{\text {final }}^{(B)}-S_{\text {initial }}^{(B)}\right] \cdot\left[\frac{d\langle C\rangle}{d S}\right]_{\text {initial }} \\
& \geq \frac{k}{\lambda} \ln 2
\end{aligned}
$$

Combining now the inequalities (19) and (21), one readily obtains a generalized version of Landauer's principle:

$$
\Delta\langle C\rangle(\lambda)=\int\left\{F_{\text {final }}^{(B)}-F_{\text {initial }}^{(B)}\right\} C(\boldsymbol{y}) d \Omega^{(B)} \geq \frac{1}{\lambda} k \ln 2
$$

which relates the initial and final mean values of the dynamical quantity $C$ associated with the environment $B$. Summing up, using (18) and basic features of the maxent probability density (which are basically those satisfied by Gibbs canonical distribution) one can derive an extension of Landauer's principle valid in the context of general divergenceless dynamical systems. This result establishes a direct link between an information-processing operation (the erasure of one bit of information from system $A$ ) and the change in the mean value of a dynamical quantity that is relevant for characterizing the state of system $B$.

\subsection{Discussion on the Derivation of the Landauer-Like Principle}

It is worth noticing that some of the assumptions made in order to obtain a Landauer-like principle for general divergenceless dynamical systems were not explicitly used in the derivation of the main result (22). Specifically, the conservation of the dynamical quantity $I$ (Equation (8)) and the hypothesis of weak interaction between systems $A$ and $B$ were not, strictly speaking, used in order to derive Equation (22). Even though these assumptions are physically very reasonable, they are not essential for obtaining (22). The precise form of the initial phase-space probability density (14) is not required either. It can be verified that a more general initial probability density of the form:

$$
F_{\text {initial }}(\boldsymbol{x}, \boldsymbol{y} ; \lambda)=F_{\text {initial }}^{(A)}(\boldsymbol{x}) \frac{e^{-\frac{\lambda}{k} C(\boldsymbol{y})}}{Z^{(B)}(\lambda)}
$$

with an arbitrary (normalizable) starting marginal probability density $F_{\text {initial }}^{(A)}$ for the system $A$ still leads to the Landauer-like result (22).

It must be emphasized that, even if the previously mentioned assumptions are not mathematically indispensable for the derivation of (22), they are physically reasonable and, in particular, lend plausibility to the initial phase-space probability density (14) as a maxent description of an equilibrium situation.

\section{Systems Described by Non-Exponential Distributions}

An interesting proposal for the description of non-equilibrium, meta-stable states has been advanced by Beck and Cohen (BC) [35-38]. The BC approach is based on the representation of statistical ensemble distributions as superpositions of Gibbs distributions characterized by different temperatures. The 
corresponding formalism is usually referred to as "superstatistics" and proven to be successful in dealing with various physical scenarios, most notably turbulence [37]. We are now going to use the BC procedure to obtain an extended Landauer-like principle for divergenceless systems described by non-exponential distributions associated with out of equilibrium situations.

In order to develop the aforementioned extension of Landauer's principle one needs a proper representation of statistical ensembles that are more general than the standard, exponential maxent ensembles. One may consider maximum entropy representations of probability distributions based on generalized entropic measures. This approach has been adopted by several researchers in recent years, with remarkable success in the modelling of different systems in meta-stable states. Alas, this procedure does not seem to be appropriate for the generalization of Landauer's principle because the physically most relevant, generalized entropies are not sub-additive. To overcome this difficulty we here apply the aforementioned alternative description of non-equilibrium ensembles, namely the one based on the BC formalism [35, 36]. This approach has the important advantage of being independent of specific properties of generalized entropic measures. Moreover, the $\mathrm{BC}$ formalism still allows for exploiting some of the nice and familiar features of the conventional Boltzmann-Gibbs-Shannon logarithmic entropy and of the associated canonical formalism. We introduce a normalized $\mathrm{BC}$ representation of the initial distribution of the composite system $A+B$ as

$$
F_{\text {initial }}(\boldsymbol{x}, \boldsymbol{y})=\frac{\int_{0}^{\infty} f(\lambda)\left[\frac{e^{-\frac{\lambda}{k}(D(\boldsymbol{x})+C(\boldsymbol{y}))}}{Z^{(A)}(\lambda) Z^{(B)}(\lambda)}\right] d \lambda}{\int_{0}^{\infty} f(\lambda) d \lambda}
$$

A non-equilibrium phase-space probability density of the form (24) is a linear superposition of a family of exponential maxent probability densities, in which the weight corresponding to each temperature is given by the function $f(\lambda)$ - the composite bit-storing device $A$ plus environment $B$ is in a nonequilibrium state characterized by a "superposition of different temperatures" or a "fluctuating temperature" described by $f(\lambda)$. The environment (both before and after the erasure process) "selects" a particular distribution of temperatures $f(\lambda)$ for the bit-storing device. In the special case of $f(\lambda)=\delta\left(\lambda-\lambda_{0}\right)$, the usual equilibrium case ensues. Notice that, for any given weight function $f(\lambda)$, the initial probability density (24) constitutes a stationary solution of Liouville's equation if $I=D(\boldsymbol{x})+C(\boldsymbol{y})$ is a constant of motion.

The starting probability density (24) is a linear combination of canonical exponential distributions $F_{\text {initial }}(\boldsymbol{x}, \boldsymbol{y} ; \lambda)$. Due to the linearity of Liouville's equation this initial distribution (24) leads, after the erasure process is completed, to the final distribution

$$
F_{\text {final }}(\boldsymbol{x}, \boldsymbol{y})=\frac{\int_{0}^{\infty} f(\lambda) F_{\text {final }}(\boldsymbol{x}, \boldsymbol{y} ; \lambda) d \lambda}{\int_{0}^{\infty} f(\lambda) d \lambda}
$$

By combining (22) with (24) and (25) we obtain a generalized Landauer-like principle providing a lower bound for the change in $\langle C\rangle$ during the erasure process,

$$
\begin{aligned}
\Delta\langle C\rangle & =\int\left\{F_{\text {final }}^{(B)}-F_{\text {initial }}^{(B)}\right\} C(\boldsymbol{y}) d \Omega^{(B)} \\
& \geq \frac{\int_{0}^{\infty} f(\lambda) \frac{k}{\lambda} d \lambda}{\int_{0}^{\infty} f(\lambda) d \lambda} \ln 2
\end{aligned}
$$


$F_{\text {initial }}^{(B)}$ and $F_{\text {final }}^{(B)}$ are, again, the bath's initial and final marginal probability distributions, respectively.

Remarks similar as those made in the previous section apply here concerning the role played by the assumptions of weak coupling between the bit-storage system $A$ and the environment $B$ and the conservation of $C+D$. Furthermore, it can be verified that an initial phase-space probability density of the form

$$
F_{\text {initial }}(\boldsymbol{x}, \boldsymbol{y})=F_{\text {initial }}^{(A)}(\boldsymbol{x}) \frac{\int_{0}^{\infty} f(\lambda) \frac{1}{Z(\lambda)} e^{-\frac{\lambda}{k} C(\boldsymbol{y})} d \lambda}{\int_{0}^{\infty} f(\lambda) d \lambda}
$$

also leads to the Landauer-like result (26).

\section{Summary and Conclusions}

In the present effort we have developed extended Landauer-like principles valid within scenarios involving general dynamical systems exhibiting divergenceless phase-space flows.

Two fundamental features of Shannon's information measure $S[F]$ lead to these Landauer-like results:

- The conservation of $S$ under the Liouville's ensemble dynamics associated with divergenceless systems.

- The sub-additive character of $S$.

The profound links between Landauer's principle and the second law of thermodynamics [21] suggest that the present results may help to explore analogues of the second law in non-standard contexts, like the biological ones discussed in [26, 27].

The lack of sub-additivity exhibited by some important non-logarithmic information or entropic functionals seems to be a serious difficulty for deriving generalizations of Landauer's principle in terms of the non-standard maxent formalisms that are nowadays popular for the study of non-equilibrium, meta-stable states. On the other hand, the Beck-Cohen approach allows for the extension of Landauer's principle to some of those scenarios. This important issue, however, needs further exploration. In this regard, any new developments towards a valid formulation of Landauer-like principles directly based upon generalized, non-standard entropic measures are very welcome.

\section{Acknowledgements}

The financial assistance of the National Research Foundation (NRF; South African Agency) towards this research is hereby acknowledged. Opinions expressed and conclusions arrived at, are those of the authors and are not necessarily to be attributed to NRF. This work was partially supported by the Projects FQM-2445 and FQM-207 of the Junta de Andalucia (Spain) and by the MEC grant No. FIS2005-02796 (Spain).

\section{References}

1. Complexity, Entropy, and the Physics of Information; Zurek, W.H., Ed.; Addison-Wesley: Redwood City, CA, USA, 1990. 
2. Beck, C.; Schlogl, F. Thermodynamics of Chaotic Systems; Cambridge University Press: Cambridge, UK, 1993.

3. Frieden, B.R.; Soffer, B.H. Lagrangians of physics and the game of Fisher-information transfer. Phys. Rev. E 1995, 52, 2274-2286.

4. Frieden, B.R. Physics from Fisher Information; Cambridge University Press: Cambridge, UK, 1998.

5. Frieden, B.R. Science from Fisher Information; Cambridge University Press: Cambridge, UK, 2004.

6. Maxwell's Demons 2: Entropy, Classical and Quantum Information; Leff, H.S., Rex, A.F., Eds.; Institute of Physics Publishing: Bristol, PA, USA, 2003.

7. Daffertshofer, A.; Plastino, A.R.; Plastino, A. Classical no-cloning theorem. Phys. Rev. Lett. 2002, $88,210601$.

8. Plastino, A.R.; Daffertshofer, A. Liouville dynamics and the conservation of classical information. Phys. Rev. Lett. 2004, 93, 138701.

9. Frieden, B.R.; Soffer, B.H. Information-theoretic significance of the Wigner distribution. Phys. Rev. A 2006, 74, 052108.

10. Hall, M.J.W. Universal geometric approach to uncertainty, entropy, and information. Phys. Rev. A 1999, 59, 2602-2615.

11. Zander, C.; Plastino, A.R.; Plastino, A.; Casas, M. Entanglement and the speed of evolution of multi-partite quantum systems. J. Phys. A: Math. Theor. 2007, 40, 2861-2872.

12. Jaynes, E.T. Papers on Probability, Statistics and Statistical Physics; Rosenkrantz, R.D., Ed.; Dordrecht: Reidel, Netherlands, 1987.

13. Plastino, A.R.; Plastino, A. Maximum entropy and approximate descriptions of pure states. Phys. Lett. A 1993, 181, 446-449.

14. Plastino, A.R.; Miller, H.G.; Plastino, A. Minimum Kullback entropy approach to the FokkerPlanck equation. Phys. Rev. E 1997, 56, 3927-3934.

15. Loewenstein, W.R. The Touchstone of Life: Molecular Information, Cell Communication, and the Foundations of Life; Oxford University Press: New York, NY, USA, 1999.

16. Lloyd, S. Rolf Landauer: Head and heart of the physics of information. Nature 1999, 400, 720.

17. Landauer, R. Irreversibility and heat generation in the computing process. IBM J. Res. Develop. 1961, 5, 183-191.

18. Piechocinska, B. Information erasure. Phys. Rev. A 2000, 61, 062314.

19. Daffertshofer, A.; Plastino, A.R. Landauer's principle and the conservation of information. Phys. Lett. A 2005, 342, 213-216.

20. Curilef, S.; Plastino, A.R.; Wedemann, R.S.; Daffertshofer, A. Landauer's principle and nonequilibrium statistical ensembles. Phys. Lett. A 2008, 372, 2341-2345.

21. Plenio, M.B.; Vitelli, V. The physics of forgetting: Landauers erasure principle and information theory. Contemp. Phys. 2001, 42, 25.

22. Daffertshofer, A.; Plastino, A.R. Forgetting and gravitation: From Landauer's principle to Tolman's temperature. Phys. Lett. A 2007, 362, 243-245.

23. Smale, S. Nonlinear Dynamics; Annals of New York Academy of Sciences 357, New York Academy of Sciences: New York, NY, USA, 1980.

24. Plastino, A.R.; Plastino, A.; da Silva, L.R.; Casas, M. Dynamical thermostatting, divergenceless 
phase-space flows, and KBB systems. Physica A 1999, 271, 343-356.

25. Roston, G.; Plastino, A.R.; Casas, M.; Plastino, A.; da Silva, L.R. Dynamical thermostatting and statistical ensembles. Eur. Phys. J. B 2005, 48, 87-93.

26. Kerner, E.H. Gibbs Ensemble, Biological Ensemble; Gordon and Breach: London, UK, 1972.

27. Kerner, E.H. Note on Hamiltonian format of Lotka-Volterra dynamics. Phys. Lett. A 1990, 151, 401-402.

28. Nambu, Y. Generalized hamiltonian dynamics. Phys. Rev. D 1973, 7, 2405-2412.

29. Bialynicki-Birula, I.; Morrison, P. Quantum mechanics as a generalization of Nambu dynamics to the Weyl-Wigner formalism. Phys. Lett. A 1991, 158, 453-457.

30. Codriansky, S.; Bernardo, C.; Aglaee, A.; Carrillo, F.; Castellanos, J.; Pereira, G.; Perez, J. Developments in Nambu mechanics. J. Phys. A 1994, 27, 2565-2578.

31. Plastino, A.R.; Plastino, A. Statistical treatment of autonomous systems with divergencelless flows. Physica A 1996, 232, 458-476.

32. Yamaleev, R.M. Generalized lorentz-force equations. Ann. Phys. N. Y. 2001, 292, 157-178.

33. Guha, P. Applications of Nambu mechanics to systems of hydrodynamical type. J. Math. Phys-NY 2002, 43, 4035.

34. Wehrl, A. General properties of entropy. Rev. Mod. Phys. 1978, 50, 221-260.

35. Beck, C.; Cohen, E.G.D. Superstatistics. Physica A 2003, 322, 267-275.

36. Beck, C.; Cohen, E.G.D. Superstatistical generalization of the work fluctuation theorem. Physica A 2004, 344, 393-402.

37. Beck, C. Statistics of three-dimensional lagrangian turbulence. Phys. Rev. Lett. 2007, 98, 064502.

38. Yamano, T. Thermodynamical and informational structure of superstatistics. Prog. Theor. Phys. Suppl. 2006, 162, 87-96.

(c) 2009 by the authors; licensee Molecular Diversity Preservation International, Basel, Switzerland. This article is an open-access article distributed under the terms and conditions of the Creative Commons Attribution license http://creativecommons.org/licenses/by/3.0/. 